Artículo

\title{
Caracterización tecnológica de las unidades de producción de tomate bajo invernadero en Puebla
}

\author{
Misael Mundo Coxca \\ José Luis Jaramillo Villanueva ${ }^{\S}$ \\ Juan Morales Jiménez \\ Antonio Macías López \\ Juventino Ocampo Mendoza \\ Colegio de Postgraduados-Campus Puebla. Boulevard Forjadores de Puebla núm. 205, Santiago \\ Momoxpan, San Pedro Cholula, Puebla, México. CP. 72760. (mmcoxca@gmail.com; morales@ colpos.mx; \\ mantonio@colpos.mx; jocampo@colpos.mx). \\ ${ }^{\S}$ Autor para correspondencia: jaramillo@ colpos.mx.
}

\section{Resumen}

Este trabajo describe las características tecnológicas de las unidades de producción de jitomate (UPJ) (Lycopersicon esculentum Mill.) bajo invernadero y las características socioeconómicas de los productores, en cuatro municipios del estado de Puebla. Los datos se obtuvieron de una muestra de productores, con una confiabilidad de $95 \%$ y precisión $10 \%$ del valor de la media de superficie del invernadero. Se aplicaron 103 encuestas a productores en el segundo semestre de 2017. Se construyó un índice tecnológico (IT) a partir de variables de manejo tecnológico y de equipamiento. El 25\% de las UPJ utiliza niveles tecnológicos bajos, 66\% niveles medios y 9\% niveles altos. La explicación del nivel tecnológico utilizado por las UPJ se estimó con un modelo regresión Probit por intervalos. El nivel de escolaridad, la experiencia en la producción bajo invernadero y el tamaño del invernadero tienen influencia significativa $(p \leq 0.5)$. Por lo tanto, se concluye que el nivel tecnológico en las unidades de producción, así como el nivel escolar, la superficie cultivada y la experiencia en la producción bajo invernadero por parte de los productores, son factores estrechamente relacionado con la productividad del cultivo. A partir de estos resultados, se podrán desarrollar e implementar estrategias que permitan brindar asesoría y capacitación en el uso adecuado de las tecnologías y equipos necesarios para lograr altos rendimientos homogéneos en la zona.

Palabras clave: agricultura protegida, tomate, unidad de producción agrícola.

Recibido: marzo de 2020

Aceptado: junio de 2020 


\section{Introducción}

En las últimas décadas, la agricultura ha hecho uso de nuevas tecnologías, principalmente plásticos que, combinados con el conocimiento en la producción de cultivos, han permitido una nueva forma de producir, conocida como agricultura protegida (AP) (Bastida, 2011).

La AP está en crecimiento, en 1980 México contaba con 300 ha, en 2008 con 10000 ha y en 2017 con 42215 ha. De estas, $39 \%$ correspondió a malla sombra. $45 \%$ a invernaderos y $16 \%$ restante a micro túnel, macro túnel y pabellón (SIAP, 2020). Sin embargo, la Encuesta Nacional Agropecuaria (INEGI, 2018) reveló que en 2016 existía 57928 ha de AP y de estas, 13694 ha eran invernaderos. La producción nacional en AP ascendió a 3.2 millones de toneladas en 2016 (INEGI, 2018). El cultivo de jitomate (Lycopersicon esculentum Mill.) en AP representa $70 \%$ de la superficie cultivada.

En el estado de Puebla se tenían 1285 ha de AP en 2016 (SIAP, 2020). El tipo de estructura que predomina es el invernadero, con $72 \%$ de ocupación en las unidades de producción (INEGI, 2018). El valor de la producción de esta hortaliza en 2014 fue de 443 millones de pesos (SIAP, 2014). La superficie cosechada y rendimiento del cultivo en el estado ha cambiado, para 2013, había 300 ha sembradas y rendimiento promedio de $175.44 \mathrm{t} \mathrm{ha}^{-1}$, mientras que en 2014 se registraron $359.46 \mathrm{ha}$ con rendimiento medio de $169 \mathrm{t} \mathrm{ha}^{-1}$ (SIAP, 2014).

En la producción de jitomate bajo invernadero, la tecnología es un componente muy importante para mejorar la productividad y los rendimientos (Padilla et al., 2010). Para abordar de forma integral el análisis de la producción de jitomate en invernadero es necesario enfocarse en la tecnología y su relación con la productividad. La tecnología es un conjunto de técnicas que permiten el aprovechamiento práctico del conocimiento científico (Burge-Smani y Wheelwright, 2004; Real Academia Española, 2018). La tecnología significa la aplicación sistemática del conocimiento científico u otro conocimiento organizado a tareas prácticas (Galbraith, 1980).

Por su parte, la productividad es considerada como la forma de utilizar los factores de producción en la generación de bienes y servicios para obtener beneficios y se resume como el cociente entre los productos generados y los insumos productivos empleados (Fontalvo-Herrera et al., 2017; Valbuena et al., 2018). La productividad en invernadero puede aumentar a través de la incorporación de mejores tecnologías.

De acuerdo con Díaz et al. (2018) las diferencias en productividad, entre las unidades de producción bajo invernadero pueden explicarse por el nivel de tecnología que utilizan en el proceso de producción. En este sentido, Ortiz et al. (2013) encontraron que a mayor adopción de tecnología, mayor es la productividad, expresado en mayor rendimiento e ingreso de las unidades productivas.

Cabe destacar que, en las unidades de producción existen diversos niveles tecnológicos que están estrechamente relacionados con el manejo del cultivo, entendido este, como las diversas actividades que el cultivo requiere. Estas actividades incluyen la preparación de la tierra, siembra, fertilización, labores culturales, control y manejo de plagas y enfermedades y la cosecha. Lo anterior, considerando el tiempo óptimo para la ejecución adecuada de técnicas y labores que el cultivo del jitomate requiere para su producción, optimizando el uso de los insumos y recursos disponibles (FAO, 2010; INIA, 2017). 
Para recomendar el uso de tecnología y el tipo de manejo que las unidades de producción deben realizar es fundamental la caracterización y estratificación de las unidades de producción. Esto permitirá formar grupos relativamente homogéneos, para la aplicación de recomendaciones de manejo eficiente del cultivo (Muñoz et al., 2015). La clasificación de las unidades de producción debe hacerse considerando el nivel tecnológico (Hernández-Ruíz et al., 2018). Sobre lo anterior, Levitan y Werneke (1984) mencionan que la tecnología y la educación permiten generar diferencias en la productividad a través del tiempo.

La caracterización tecnológica de la producción de jitomate permitirá comprender la dinámica y funcionamiento del cultivo en el contexto de su desarrollo, evolución y cambio con la finalidad de lograr planes y programas para grupos con características similares (Valerio et al., 2004). Por lo anterior, esta investigación tiene por objetivo caracterizar el nivel tecnológico de las unidades de producción de jitomate (UPJ) de cuatro municipios de Puebla e identificar las variables sociodemográficas y económicas que explican el comportamiento tecnológico.

\section{Materiales y métodos}

\section{Localización de la zona de estudio}

Aquixtla y Tetela de Ocampo se ubican en la parte norte del estado de Puebla, entre los 1200 y los $3200 \mathrm{msnm}$. El clima de estos municipios es del tipo templado subhúmedo con temperaturas van de los 12 a los $20^{\circ} \mathrm{C}$. La precipitación varía de 600 a $1600 \mathrm{~mm}$. Aquixtla tiene 7386 habitantes y Tetela de Ocampo 24459 habitantes (INEGI, 2009). Por su parte, Tecamachalco y Tochtepec se ubican en el centro del estado de Puebla, presentan un clima templado subhúmedo con lluvias en verano (100\%), con temperaturas que oscilan entre 14 y $18{ }^{\circ} \mathrm{C}$ y una precipitación de 500 a 700 mm. Tecamachalco tiene 64380 habitantes y Tochtepec 18205 habitantes (INEGI, 2009).

La población total considerada fueron 338 productores bajo invernadero en el estado de Puebla registrados en el sistema nacional de información de agricultura protegida (SIAPRO, 2011), el cual permitió localizar los municipios con el mayor número de unidades. La población objetivo del estudio fueron los productores de los municipios de Aquixtla, Tetela de Ocampo, Tecamachalco y Tochtepec que representan $43.5 \%$ de la superficie de invernaderos con jitomate en el estado de Puebla (SIAP, 2014).

Se utilizó un muestreo simple aleatorio. El tamaño de muestra de 103 productores fue calculado con muestreo aleatorio simple, con precisión de $10 \%$ de la media de la superficie de invernaderos en el estado y confiabilidad del cinco por ciento.

La unidad de análisis en el estudio, UPJ, representa la unidad económica que ocupa un determinado conjunto de terreno(s), infraestructura, maquinaria y equipo. que son utilizados durante las actividades agropecuarias y no agropecuarias por el grupo familiar. Este grupo familiar se rige bajo una misma administración, en la cual prevalecen determinadas relaciones de diseño, organización, toma y ejecución de decisiones (Martínez et al., 2014).

Los datos se obtuvieron a través de un cuestionario aplicado directamente al productor en el segundo semestre de 2017. Dicho cuestionario estuvo integrado por 177 preguntas, divididas en: a) aspectos sociodemográficos del productor; b) tenencia de la tierra y experiencia en producción; 
c) aspectos técnicos del invernadero; d) aspectos de producción (siembra, preparación del suelo, labores culturales y nutrición); y e) equipos adicionales en el invernadero. Con estos datos se construyó un índice tecnológico (IT), para representar el nivel tecnológico de cada UPJ. El IT estuvo integrado por dos subíndices, el índice de manejo tecnológico (IMT) (Cuadro 1A y 1B) y el índice de equipamiento (IEQ) (Cuadro 2).

Cuadro 1A. Componentes del índice de manejo tecnológico.

\begin{tabular}{|c|c|c|}
\hline $\mathrm{CAT}^{*}$ & Tipo & Valor** \\
\hline \multirow[t]{3}{*}{ Material genético } & Híbrido & 1 \\
\hline & Variedad & 2 \\
\hline & Variedad-híbrido & 3 \\
\hline \multirow[t]{3}{*}{ Edad al trasplante } & 30-50 días & 1 \\
\hline & 20-30 días & 2 \\
\hline & 10-20 días & 3 \\
\hline \multirow{3}{*}{$\begin{array}{l}\text { Número de plantas por } \\
\text { metro cuadrado }\end{array}$} & De 1 a 4 plantas $\mathrm{m}^{-2}$ & 1 \\
\hline & De 5 a 8 plantas $\mathrm{m}^{-2}$ & 2 \\
\hline & De 9 a 12 plantas $m^{-2}$ & 3 \\
\hline \multirow[t]{3}{*}{ Frecuencia de riego } & Cada tercer día & 1 \\
\hline & Diario & 2 \\
\hline & De acuerdo con las necesidades de la planta & 3 \\
\hline \multirow[t]{3}{*}{ Poda de tallos } & De tres a más tallos & 1 \\
\hline & A un tallo & 2 \\
\hline & A dos tallos & 3 \\
\hline \multirow[t]{3}{*}{ Forma de polinizar } & Vibración manual de la planta (paleo y sacudiendo hilos) & 1 \\
\hline & Alternancia entre vibración manual y abejorros & 2 \\
\hline & $\begin{array}{l}\text { Uso único de abejorros y pulverizador de aire para } \\
\text { polinizar }\end{array}$ & 3 \\
\hline
\end{tabular}

Cuadro 1B. Componentes del índice de manejo tecnológico.

\begin{tabular}{llc}
\hline \multicolumn{1}{c}{ CAT $^{*}$} & \multicolumn{1}{c}{ Tipo } & Valor $^{* *}$ \\
\hline Altura de trasplante & Menos de 15 y más de $26 \mathrm{~cm}$ & 1 \\
& De 21 a $25 \mathrm{~cm}$ & 2 \\
& De 15 a $20 \mathrm{~cm}$ & 3 \\
Poda de brotes, hojas & Solo poda de brotes o solo poda de hojas o solo raleo & 1 \\
y raleo & Poda de hojas y brotes o poda de hojas y raleo o podas de brotes & 2 \\
& y raleo & 3 \\
\hline
\end{tabular}




\begin{tabular}{llc}
\hline \multicolumn{1}{c}{ CAT $^{*}$} & \multicolumn{1}{c}{ Tipo } & Valor $^{* *}$ \\
\hline $\begin{array}{l}\text { Número de podas } \\
\text { realizadas en el ciclo }\end{array}$ & Menos de 20 podas & 1 \\
& De 20 a 24 & 2 \\
Suelo & Más de 24 & 3 \\
& $\begin{array}{l}\text { Acolchado dentro de las instalaciones o solo desinfección de } \\
\text { suelo o solo aplicación de abonos previos a la siembra }\end{array}$ & 1 \\
& Acolchado de planta y desinfección o acolchado y aplicación de & 2 \\
& $\begin{array}{l}\text { abono previo o desinfección de suelo y aplicación de abono } \\
\text { previo }\end{array}$ & \\
& Acolchado de planta, desinfección de suelo y aplicación de & 3 \\
& abono previo a la siembra & \\
\hline
\end{tabular}

*= componente y actividad tecnológica; ${ }^{* *}=$ valor asignado.

Cuadro 2. Componentes del índice de equipamiento.

\begin{tabular}{lc}
\hline Equipo & Valor \\
\hline Báscula & 1 \\
Mochila aspersora manual & 1 \\
Termómetro de mercurio & 1 \\
Termómetro digital (higrómetro) & 2 \\
Higrómetro de suelo & 2 \\
Conductímetro & 2 \\
Peachímetro & 2 \\
Parihuela & 3 \\
Mochila aspersora de motor & 3 \\
Quemador de gas & 4 \\
Computadora & 4 \\
Riego computarizado & 4 \\
Calefactor con ventilador & 4 \\
\hline
\end{tabular}

Elaboración con base a García et al. (2011), modificado para la zona de estudio.

\section{Índice de manejo tecnológico}

Este índice se concentra en las labores culturales realizadas por los productores, dado que no existe la referencia de un paquete de manejo tecnológico validado por alguna entidad gubernamental o de investigación en la zona de estudio.

Para su construcción se tomó en cuenta el índice propuesto por Ortiz (2005) en función a las labores culturales realizadas a la planta y la tecnología empleada. La propuesta de Ortiz (2005) tuvo adecuaciones de Carrillo et al. (2003) en el componente de densidad de siembra, de Martínez et al. (2005) en material genético utilizado y de Ucan et al. (2005) en los componentes de podas, raleo y 
riego. Además, en la construcción del Índice se incorporó la opinión de productores líderes en la zona de estudio en el contexto del manejo del cultivo, asignando un valor a cada componente (mínimo (1), intermedio (2) y máximo (3)) dentro de las labores realizadas. Para el análisis del IMT se aplicó la siguiente formula: IMT= X - Min/Max - Min.

Donde: IMT= es el índice de manejo tecnológico; $\mathrm{X}=$ es la $\sum$ de los valores acumulados de las 10 prácticas o labores culturales que realizan los productores de jitomate; Min= es el valor mínimo de los puntos acumulados para cada caso igual a 10; y $\mathrm{Max}=$ es el valor máximo de los puntos acumulados igual a 30 .

\section{Índice de equipamiento}

El IEQ se construyó asignando un valor al número de equipos adicionales al sistema de riego en los invernaderos. Se estableció un rango de valores conforme la importancia y grado de complejidad de cada equipo, siendo el máximo 4 y el mínimo 1 (García et al., 2011). Para su cálculo se aplicó la siguiente formula: IEQ= X - Min/Max - Min.

Donde: $\mathrm{IEQ}=$ es el nivel de equipamiento; $\mathrm{X}=$ es la $\sum$ de los valores acumulados de los 13 equipos adicionales al sistema de riego en el invernadero; Min= es el valor mínimo de los puntos acumulados para cada caso igual a 1 y $\mathrm{Max}=$ es el valor máximo de los puntos acumulados igual a 33 .

Una vez obtenido el índice de equipamiento de las UPJ, se describió su frecuencia y porcentaje de presencia en la zona de estudio.

\section{Índice tecnológico}

El IT es el promedio aritmético del valor del IMT y del IEQ. Esto debido a que, en opinión de expertos (agrónomos especialistas en la producción de tomate en invernadero), ambos son determinantes para la productividad. Para explicar el comportamiento del IT se utilizó un modelo de regresión Probit ordenado. Este modelo permite el cálculo de las probabilidades predichas para cada categoría de la variable dependiente y sus efectos marginales.

El modelo Probit tiene una distribución simétrica en forma de campana (Greene, 2008). El efecto marginal significa que un cambio de una unidad en la variable explicativa dará lugar a un aumento o disminución en la probabilidad prevista igual al tamaño del efecto marginal. La elección de este modelo se debe a las ventajas que representa en su forma funcional pues las probabilidades estimadas están en el intervalo 0-1 y permite captar efectos no lineales de las variables explicativas sobre la variable explicada (Long, 1997).

Las variables con las que se estimó el modelo empírico se describen en el Cuadro 3. La especificación es la siguiente:

$$
\begin{aligned}
& I T i={ }_{0}+{ }_{1} E D A D 1+{ }_{2} E D A D 2+{ }_{3} E S C 1+{ }_{4} E S C 2+{ }_{5} E S C 3+{ }_{6} E S C 4+{ }_{7} E X P 1+{ }_{8} E X P 2+{ }_{9} E X P 2+{ }_{10} E X P I 1+ \\
& +{ }_{11} E X P I 2+{ }_{12} E X P I 3+{ }_{13} R E N 1+{ }_{14} R E N 2+{ }_{15} R E N 3+{ }_{16} S U P 1+{ }_{17} S U P 2+{ }_{18} S U P 3+{ }_{19} S U P 4+{ }_{i}
\end{aligned}
$$


Cuadro 3. Variables utilizadas en el modelo Probit.

\begin{tabular}{|c|c|c|}
\hline Variable original & Variable categórica & $\begin{array}{c}\text { Código de variable } \\
\text { categórica }\end{array}$ \\
\hline \multirow[t]{2}{*}{ Edad del productor } & 1) Menor a 35 años (EDAD1) & Edad 1 \\
\hline & 2) De 36 a 55 años (EDAD1) & Edad 2 \\
\hline \multirow[t]{4}{*}{ Escolaridad } & 3) Primaria (ESCOL1) & Escol 1 \\
\hline & 4) Secundaria (ESCOL2) & Escol 2 \\
\hline & 5) Preparatoria (ESCOL3) & Escol 3 \\
\hline & 6) Licenciatura y posgrado (ESCOL4) & Escol 4 \\
\hline \multirow{3}{*}{$\begin{array}{l}\text { Años de experiencia como } \\
\text { agricultor }\end{array}$} & 7) De 6 a 10 años (EXP1) & $\operatorname{Exp} \mathrm{A} 1$ \\
\hline & 8) De 11 a 20 años (EXP2) & $\operatorname{Exp} A 2$ \\
\hline & 9) Más de 20 años (EXP3) & $\operatorname{Exp} A 3$ \\
\hline \multirow{3}{*}{$\begin{array}{l}\text { Años de experiencia en } \\
\text { producción bajo invernadero }\end{array}$} & 10) De 3 a 6 años (EXPI1) & Exp I1 \\
\hline & 11) De 7 a 9 años (EXPI2) & Exp I2 \\
\hline & 12) Más de 10 años (EXPI3) & Exp I3 \\
\hline \multirow[t]{3}{*}{ Rendimiento de la UPJ } & 13) De 101 a $150 \mathrm{t} \mathrm{ha}^{-1}$ (REN1) & Rendim 1 \\
\hline & 14) De 151 a $300 \mathrm{t} \mathrm{ha}^{-1}$ (REN2) & Rendim 2 \\
\hline & 15) Más de 300 t ha'-1 (REN3) & Rendim 3 \\
\hline \multirow[t]{4}{*}{ Superficie de la UPJ } & 16) 1001 a $2000 \mathrm{~m}^{2}$ (SUP1) & Sup 1 \\
\hline & 17) 2001 a $3000 \mathrm{~m}^{2}$ (SUP2) & Sup 2 \\
\hline & 18) 3001 a $5000 \mathrm{~m}^{2}$ (SUP3) & Sup 3 \\
\hline & 19) 5000 m² o más (SUP4) & Sup 4 \\
\hline
\end{tabular}

Encuestas aplicadas en julio 2017.

La variable dependiente es ITi, con tres diferentes categorías IMT=0; IMT= 1 (bajo); IMT= 2 (medio); IMT = 3 (alto), los rangos fueron definidos conforme a los resultados obtenidos en la creación del índice, $\beta_{0}=$ constante de la regresión, $\beta i=$ coeficientes estimados de la regresión.

\section{Resultados y discusión}

El $92 \%$ de los productores de jitomate bajo invernadero son hombres y $8 \%$ son mujeres. Este porcentaje de hombres es mayor al reportado por Martínez et al. (2014). La edad promedio fue de 42 años, similar a lo reportado por Vargas et al. (2015). El promedio de escolaridad es de 8.6 años, mayor a los 6 años reportados por Ortega et al. (2014) para productores de jitomate y cercano a Vargas et al. (2015) de 9.22 años. La producción de jitomate en invernadero es la fuente principal de ingresos para $77 \%$ de los productores, mientras que $23 \%$ realiza una combinación de actividades agropecuarias y no agropecuarias.

El $70 \%$ del agua utilizada en el cultivo proviene de ríos y manantiales, dato similar al reportado por Ortega et al. (2014). El otro 30\% del agua se obtiene de pozos. Los invernaderos de multitúnel con ventilación del tipo cenital móvil y fija representan el tipo de construcción más frecuente 
(94\%), con una superficie promedio de $3900 \mathrm{~m}^{2}$, lo que es mayor a lo reportado por Ortega et al. (2014), Vargas et al. (2015) y Martínez et al. (2014). Los materiales utilizados en la estructura del invernadero son el perfil tubular rectangular (PTR) galvanizado y las cubiertas de plásticos de 16 diferentes variantes. El 100\% de las construcciones emplea el polietileno como material de cubierta (Martínez et al., 2014; Ortega et al., 2014). De las UPJ estudiadas, 43\% se establecieron con recursos propios, $30 \%$ con apoyo gubernamental (Ortega et al., 2014) y 27\% con créditos. Sin embargo, Martínez et al. (2014) reportó que 77.6\% de los invernaderos se establecieron con inversión federal o estatal y el restante $22.4 \%$ con recursos propios.

El funcionamiento de los invernaderos tiene 7 años en promedio, cifra mayor a la reportado por Martínez et al. (2014); Ortega et al. (2014); Vargas et al. (2015). Existen dos ciclos de siembra, enero-julio y septiembre-febrero. La cosecha se realiza en mayo-julio y octubre-diciembre. La siembra se lleva a cabo mediante semilla (66\%) y esqueje (34\%). Se identificó 23 variedades diferentes utilizadas en los últimos dos ciclos de siembra. Las variedades más utilizadas son: reserva, El Cid, Pai-Pai, Saladette y Optimax con 65\%. El 35\% restante lo integran 18 variedades diferentes a las mencionadas anteriormente. El número promedio de plantas por $\mathrm{m}^{2}$ fue de 3.1 (Martínez et al., 2014).

El rendimiento promedio es de $152 \mathrm{t} \mathrm{ha}^{-1}, 17 \mathrm{t}$ por debajo del promedio estatal y 22 menos que el promedio nacional. Estos rendimientos difieren de lo observado por Vargas et al. (2015) y Ortega et al. (2014). Las ventas se realizaron en su mayoría a intermediarios (74\%) que colectaron el producto directamente en las UPJ. El resto fueron ventas hechas a la central de abastos (23\%) de Puebla, México, Morelos y Toluca. Sólo 3\% de las ventas se hizo a mercados locales. Al respecto, Hernández-Ruíz et al. (2018) reportaron que la comercialización por pequeños productores se realiza fundamentalmente en el mercado regional y local.

La mano de obra utilizada es de tipo familiar en el $70 \%$ de las UPJ, similar a lo reportado por Martínez et al. (2014). El resto de las UPJ contrataron al menos cuatro personas para realizar las actividades diarias en el invernadero. Los productores que han recibido capacitación y asistencia técnica ascienden a $35 \%$. Adicionalmente $13 \%$ de los productores se han capacitado en aspectos de comercialización. Este aspecto es de importancia debido a que la capacitación de quienes se encargan del manejo y administración de la UPJ tiene influencia positiva en su productividad (Moreno et al., 2011). La asesoría en aspectos de comercialización ha sido brindada (en orden de importancia) por el gobierno estatal e instituciones educativas de nivel superior del estado de Puebla. Esto difiere de lo reportado por Ortega et al. (2014), que destaca la nula relación de los productores con algún centro de investigación o universidad.

\section{Índice de manejo tecnológico (IMT)}

Conforme a los resultados encontrados en el análisis de las 10 variables categóricas que integran los componentes tecnológicos y labores culturales (IMT) se obtuvieron valores que van de 0.15 a 0.70 , permitiendo determinar 3 diferentes niveles; menor a $0.25=$ bajo; de 0.26 a $0.5=$ medio y mayor a $0.5=$ alto. El $60 \%$ de las UPJ cuentan con un nivel tecnológico medio, $26 \%$ bajo y $14 \%$ alto. 


\section{Índice de equipamiento (IEQ)}

El IEQ de los invernaderos mostró puntajes que van de 0.06 a 0.76 , permitiendo determinar 3 diferentes niveles: menor a $0.25=$ bajo; de 0.26 a $0.5=$ medio y mayor a $0.5=$ alto. Las UPJ con nivel bajo representó el 25\%, con nivel medio el $66 \%$ y con nivel alto el 9\%. En el Cuadro 4, se aprecian las diferencias de rendimiento conforme al nivel en cada uno de los índices.

Cuadro 4. Número de productores y promedio de rendimiento en cada índice obtenido.

\begin{tabular}{|c|c|c|c|c|c|c|}
\hline \multirow{2}{*}{ Índices obtenidos } & \multicolumn{2}{|r|}{ Bajo } & \multicolumn{2}{|r|}{ Medio } & \multicolumn{2}{|r|}{ Alto } \\
\hline & $\mathrm{n}$ & Rendimiento* & $\mathrm{n}$ & Rendimiento* & $\mathrm{n}$ & Rendimiento* \\
\hline IMT & 27 & 91.63 & 62 & 152.7 & 14 & 263.2 \\
\hline IEQ & 40 & 128.7 & 57 & 156 & 6 & 264.5 \\
\hline
\end{tabular}

${ }^{*}=$ rendimiento promedio.

Conforme a los resultados anteriores, se deduce que el número de equipos adicionales y las actividades aplicadas en el cultivo permiten incrementar los rendimientos. Resultados similares fueron reportados por García et al. (2011) quien, en un estudio sobre adopción de innovaciones en Tlaxcala encontró una relación positiva entre nivel de equipamiento y rendimientos.

\section{Análisis del índice tecnológico}

El IT representa el nivel de uso de tecnología de cada unidad de producción, el cual está relacionado con el rendimiento de jitomate; la diferencia de las medias de rendimiento del IT bajo-medio, medio-alto y bajo-alto son significativos $(p \leq 0.05)$ (Cuadro 5).

Cuadro 5. Diferencia de las medias del rendimiento entre las categorías del IT.

\begin{tabular}{cccccc}
\hline Nivel del IT & Observaciones & Media de rendimiento & Dif. de la media & Mínimo & Máximo \\
\hline Bajo & 26 & 98.6 & - & 34 & 180 \\
Medio & 68 & 158.4 & $59.8^{*}$ & 24 & 340 \\
Alto & 9 & 255 & $96.6^{*}$ & 200 & 360 \\
Total & 103 & 151.7 & - & 24 & 360 \\
\hline
\end{tabular}

* $=$ la diferencia de las medias es significativa $(p \leq 0.05)$.

Para explicar el comportamiento del IT se estimó un modelo de regresión Probit. Las estimaciones de los parámetros y las estadísticas de ajuste del modelo se muestran en el Cuadro 6. Siete variables en el modelo fueron significativas $(p<0.05)$. Estas fueron la escolaridad en el estrato que integran los productores con licenciatura o posgrado (Águila y Padilla, 2010; Vargas et al., 2015), la experiencia como agricultor en el estrato de 11 a 20 años, la experiencia con la agricultura bajo invernadero, el estrato de más de 10 años, y la superficie del invernadero en los estratos 3001 a 5 $000 \mathrm{~m}^{2}$ y más de $5000 \mathrm{~m}^{2}$. Sobre esta última variable Hernández-Ruíz et al. (2018), en un estudio realizado en Oaxaca, identificó una relación positiva entre el nivel tecnológico, rendimiento y tamaño del invernadero. 
Cuadro 6. Resultados del modelo Probit del IT.

\begin{tabular}{|c|c|c|c|c|}
\hline Variable código & Variable & $\beta$ & Error estándar & Valor de $\mathrm{t}$ \\
\hline Edad 1 & Menor de 35 años & -0.32 & 0.75 & -0.43 \\
\hline Edad 2 & De 36 a 55 años & 0.41 & 0.66 & 0.62 \\
\hline Escol 1 & Primaria & 1.36 & 0.89 & 1.53 \\
\hline Escol 2 & Secundaria & 1.55 & 0.83 & $1.87^{*}$ \\
\hline Escol 3 & Preparatoria & 1.19 & 0.85 & 1.4 \\
\hline Escol 4 & Licenciatura y posgrado & 4.89 & 1.46 & $3.35^{* *}$ \\
\hline Exp A1 & De 6 a 10 años & -0.88 & 0.58 & -1.52 \\
\hline $\operatorname{Exp} A 2$ & De 11 a 20 años & -0.75 & 0.31 & $-2.42^{* *}$ \\
\hline Exp A3 & Más de 20 años & -0.78 & 0.6 & -1.3 \\
\hline Exp I1 & De 3 a 6 años & 0.74 & 0.61 & 1.21 \\
\hline Exp I2 & De 7 a 9 años & 0.22 & 0.7 & 0.31 \\
\hline Exp I3 & Más de 10 años & 0.73 & 0.2 & $3.65^{* *}$ \\
\hline Rendim 1 & De 101 a $150 \mathrm{tha}^{-1}$ & 0.4 & 0.42 & 0.95 \\
\hline Rendim 2 & De 151 a $300 \mathrm{tha}^{-1}$ & 1.61 & 0.58 & $2.78^{* *}$ \\
\hline Rendim 3 & Más de $300 \mathrm{t} \mathrm{ha}^{-1}$ & 3.01 & 0.83 & $3.63^{* *}$ \\
\hline Sup 1 & De 1001 a $2000 \mathrm{~m}^{2}$ & 0.19 & 0.59 & 0.32 \\
\hline Sup 2 & De 2001 a $3000 \mathrm{~m}^{2}$ & -0.79 & 0.55 & -1.44 \\
\hline Sup 3 & De 3001 a $5000 \mathrm{~m}^{2}$ & 0.68 & 0.22 & $3.09^{* *}$ \\
\hline Sup 4 & De 5000 m² o más & 0.35 & 0.11 & $3.18^{* *}$ \\
\hline \multicolumn{2}{|c|}{ Log likelihood } & \multicolumn{3}{|c|}{-45.333444} \\
\hline \multicolumn{2}{|c|}{ LR chi $^{2}$} & \multicolumn{3}{|c|}{112.88} \\
\hline \multicolumn{2}{|c|}{ Prob $>\mathrm{chi}^{2}$} & \multicolumn{3}{|c|}{0} \\
\hline \multicolumn{2}{|c|}{ Pseudo $\mathrm{R}^{2}$} & \multicolumn{3}{|c|}{0.581} \\
\hline
\end{tabular}

${ }^{* *}=$ variables significativas $(p<0.05)$.

En el modelo Probit, los coeficientes del modelo se interpretan como logaritmo de la razón de probabilidades (odd ratios). Por cada incremento de una unidad en la variable explicativa, la variable respuesta (IT) cambia en alguna de sus categorías, en la magnitud del coeficiente (Greene, 2008). Para hacer más informativos los coeficientes del modelo, se calculó el efecto marginal que define el impacto de un cambio en una variable explicativa sobre las probabilidades predichas de IT, para una variable explicativa, un cambio de una unidad impacta en aumento o disminución en las probabilidades predichas, en la magnitud del efecto marginal (Greene, 2008). El Cuadro 7 presenta los efectos marginales de las variables explicativas del modelo, para cada uno de los niveles de IT. 
Cuadro 7. Efectos marginales en el modelo de IT.

\begin{tabular}{|c|c|c|c|c|}
\hline Variable código & Variable & $\begin{array}{c}\mathrm{IT}=1 \\
\text { (Bajo) }\end{array}$ & $\begin{array}{c}\text { IT=2 } \\
\text { (Medio) }\end{array}$ & $\begin{array}{l}\mathrm{IT}=3 \\
\text { (Alto) }\end{array}$ \\
\hline Edad 1 & Menor de 35 años & 0.014 & 0.016 & -0.03 \\
\hline Edad 2 & De 36 a 55 años & -0.018 & -0.020 & 0.041 \\
\hline Escol 1 & Primaria & -0.06 & -0.07 & -0.134 \\
\hline Escol 2 & Secundaria & -0.073 & -0.081 & 0.055 \\
\hline Escol 3 & Preparatoria & -0.051 & -0.062 & 0.114 \\
\hline Escol 4 & Licenciatura y posgrado & -0.231 & -0.269 & 0.499 \\
\hline $\operatorname{Exp} A 1$ & De 6 a 10 años & 0.035 & 0.045 & -0.089 \\
\hline $\operatorname{Exp} A 2$ & De 11 a 20 años & 0.053 & 0.062 & -0.118 \\
\hline Exp A3 & Más de 20 años & 0.042 & 0.045 & -0.088 \\
\hline Exp I1 & De 3 a 6 años & -0.037 & -0.045 & 0.085 \\
\hline Exp I2 & De 7 a 9 años & -0.013 & -0.015 & 0.125 \\
\hline Exp I3 & Más de 10 años & -0.062 & -0.071 & 0.13 \\
\hline Rendim 1 & De 101 a $150 \mathrm{t} \mathrm{ha}^{-1}$ & -0.136 & 0.135 & 0.002 \\
\hline Rendim 2 & De 151 a $300 \mathrm{t} \mathrm{ha}^{-1}$ & -0.349 & 0.305 & 0.539 \\
\hline Rendim 3 & Más de 300 t ha-1 & -0.371 & -0.579 & 0.945 \\
\hline Sup 1 & De 1001 a $2000 \mathrm{~m}^{2}$ & -0.005 & -0.015 & -0.018 \\
\hline Sup 2 & De 2001 a $3000 \mathrm{~m}^{2}$ & 0.071 & -0.025 & -0.045 \\
\hline Sup 3 & De 3001 a $5000 \mathrm{~m}^{2}$ & -0.002 & -0.003 & 0.113 \\
\hline Sup 4 & De $5000 \mathrm{~m}^{2}$ o más & -0.008 & -0.016 & 0.129 \\
\hline
\end{tabular}

La escolaridad está directamente relacionada con un mayor IT, tener primaria disminuye la probabilidad de tener IT alto mientras que tener preparatoria aumenta la probabilidad de tener IT alto en $11 \%$. Por su parte tener licenciatura o mayor, aumenta la probabilidad de tener IT alto en 49.9\%. Esto significa que tener capital humano capacitado, representado por el nivel de escolaridad, facilita la asimilación de tecnologías en la empresa (Águila y Padilla, 2010). Además, el nivel escolar puede ser un factor determinante en el desempeño de las tecnológicas utilizadas en la agricultura protegida (Vargas et al., 2015). Tener experiencia en la producción en invernadero también está relacionado con un mayor nivel de IT, especialmente mayor a siete años, lo que aumenta la probabilidad de IT alto en $12.5 \%$. Más allá de los 10 años de experiencia en este aspecto, no aumenta la probabilidad de un IT alto de forma importante. La superficie muestra que menos de $3000 \mathrm{~m}^{2}$ reduce las probabilidades de tener un IT alto mientras que a partir de esta superficie en invernadero, las probabilidades aumentan de forma importante.

\section{Conclusiones}

El cultivo de jitomate en muestra una actividad productiva permanente en los últimos siete años de haber sido establecidos los invernaderos. Los apoyos gubernamentales no fueron un factor importante en el establecimiento y funcionamiento de los invernaderos. Las UPJ en el estado de Puebla cuentan con niveles tecnológicos medios y bajos principalmente, siendo una determinante el IT en el rendimiento obtenido por invernadero, dado que las UPJ con un IT Alto presentan un promedio de rendimiento mayor. 
Las variables sociodemográficas y económicas más importantes que explican el nivel tecnológico, y también las que están directamente relacionadas con un IT alto son el nivel de escolaridad, la experiencia en la producción bajo invernadero, el rendimiento alto y la superficie cultivada bajo esta modalidad. Estos resultados se pueden usar para generar estrategias tendientes a mejorar la adopción de tecnología, que permitan mantener rendimientos altos.

La productividad de las UPJ radica en la experiencia del manejo del cultivo y la forma en que se realizan las actividades y componentes tecnológicos aplicados al jitomate, así como el equipamiento en cada una de ellas. Los factores anteriores son determinantes en la mejora del rendimiento. Por ello, es necesario fortalecer el vínculo de los centros de investigación y gobierno en sus distintos niveles con los productores de jitomate en el contexto de asesoramiento y capacitación continua.

\section{Literatura citada}

Águila, O. A. R. D. and Padilla, M. A. 2010. Factores determinantes de la innovación en empresas de economía social. La importancia de la formación y de la actitud estratégica. CIRIECEspaña. Rev. Econ. Pública, Social y Cooperativa. 67:129-155.

Bastida, T. A. y Ramírez, A. J. A. 2011. Los invernaderos y la agricultura protegida en México. Serie e Publicaciones Agribot. Departamento de Preparatoria Agrícola-Universidad Autónoma Chapingo (UACH). Chapingo, Estado de México. 415 p.

Burge-Smani, R. A and Wheelwright S. C. 2004. Strategic management of technology and innovation. Reading. 1(1):66-77.

Carrillo, J.; Jiménez, F.; Ruiz, J.; Díaz, G.; Sánchez, P.; Perales, C. y Arellanes, A. 2003. Evaluación de densidades de siembra en tomate (Lycopersicon esculentum Mill.) en invernadero. Agron. Mesoam. 14(1):85-88.

Díaz, R. C.; Pérez de la Cruz, J. M. y Ramos-Herrera. 2018. Nuevas y competitividad: implicaciones en una unión monetaria. Rev. Econ. Mundial. 49:39-56.

FAO. 2010. Buenas prácticas agrícolas en la cadena de tomate. Primera edición, Buenos Aires, Argentina. ISBN-978-92-5-306646-9.

Fontalvo-Herrera, T.; De La Hoz-Granadillo, E. y Morelos-Gómez, J. 2017. La productividad y sus factores: incidencia en el mejoramiento organizacional. Dimensión empresarial. 15(2):47-60. Doi: http://dx.doi.org/10.15665/rde.v15i2.1375. http://www.scielo.org.co/ pdf/ diem/ v16n1/1692-8563-diem-16-01-00047.pdf.

Galbraith, J. K. 1980. El nuevo estado industrial. Ariel. Barcelona, España. 374 p.

García, S. E. I.; Ávila, J. A. y Muñoz, R. B. 2011. La agricultura protegida en Tlaxcala. México. La adopción de innovaciones y el nivel de equipamiento como factores para su categorización. Teuken Bidikay. 2(2):193-212.

Greene, W. H. 2008. Econometric analysis. $6^{\text {th }}$ (Ed.). Upper Saddle River. Prentice Hall. $710-714$ pp.

Hernández-Ruíz, J.; Espinosa-Trujillo, E.; Míreles-Arriaga, A. y Ruiz-Nieto, J. E. 2018. Índice tecnológico de las unidades de producción de tomate en invernadero en Tlahuitoltepec, Oaxaca. Acta Agrícola y Pecuaria. 4(2):35-43.

INEGI. 2009. Instituto Nacional de Estadística y Geografía. Censo Agrícola, Ganadero y Forestal. Prontuario de información geográfica municipal de los Estados Unidos Mexicanos. Tecamachalco, Puebla. Clave geoestadística 21154. 9 p.; Tetela de Ocampo, Puebla Clave geoestadística 21172. 9 p.; Tochtepec, Puebla. Clave geoestadística 21189. 9 p.; Aquixtla, Puebla. Clave geoestadística 21016. 9 p. 
INEGI. 2018. Instituto Nacional de Estadística y Geografía. Encuesta Nacional Agropecuaria 2017. https://www.inegi.org.mx/programas/ena/2017/.

INIA. 2017. Instituto de Investigaciones Agropecuarias. Manual del cultivo del tomate bajo invernadero. Boletín INIA, No. 12, ISSN:0717-4829. 111 p.

Levitan, S. A. and Werneke, D. 1984. Productivity: problems, prospects, and policies. The Johns Hopkins University Press No. 40. 200 p.

Long, J. S. 1997. Regression models for categorical and limited dependent variables. Thousand Oaks, CA. Sage Publications. 297 p.

Martínez-Gutiérrez, G. A.; Díaz-Pichardo, R.; Juárez-Luis, G.; Ortiz-Hernández, Y. D. y LópezCruz, J. Y. 2014. Caracterización de las unidades de producción de tomate en invernaderos de Oaxaca. Agric. Soc. Des. 11(2):153-165.

Martínez-Solís, J.; Peña-Lomelí, A.; Rodríguez-Pérez, J.; Villanueva-Verduzco, C.; SahagúnCastellanos, J. y Peña-Ortega, M. 2005. Comportamiento productivo en híbridos de jitomate y sus respectivas poblaciones F2. Rev. Chapingo Ser. Hortic. 11(2):299-307.

Moreno, R.; Aguilar, D. y Luévano, G. 2011. Características de la agricultura protegida y su entorno en México. Rev. Mex. Agron. 29(2):763-774.

Muñoz-ácome, E. A.; Coello, M. J.; Moreno, F. y Cruz, C. 2015. Metodología para la evaluación del nivel tecnológico del cultivo de Rye grass en los Andes ecuatorianos, microcuenca del río Chimborazo. Rev. Iberoam. Cienc. Biol. Agrop. 4(8):88-117.

Ortega, M. L. D.; Ocampo, M. J.; Sandoval, C. E. y Martínez, V. C. 2014. Caracterización y funcionalidad de invernaderos en Chignahuapan, Puebla. Rev. Bio Cienc. 2(4):261-270.

Ortiz, N.; López, A. A.; Alejandro, G. L. \& Felipe, E. P. 2005. La tecnología en la producción de jitomate (Lycopersicon esculentum Mill.) en invernadero y su efecto socio-económico en las regiones cafetaleras de Huatusco y Córdoba, Veracruz. Colegio de PostgraduadosCampus Veracruz-Programa en Agroecosistemas Tropicales. 37-39 pp.

Ortiz-Jiménez, B.; Jiménez-Sánchez, L.; Morales-Guerra, M.; Quispe-Limaylla, A.; TurrentFernández, A.; Rendón-Sánchez, G. y Rendón-Medel, R. 2013. Nivel de adopción de tecnologías para la producción de jitomate en productores de pequeña escala en el estado de Oaxaca. Rev. Mex. Cienc. Agríc. 4(3):447-460.

Padilla, L. E.; Rumayor, A.; Pérez, O. y Reyes, E. 2010. Competitiveness of Zacatecas (México) Protected agriculture: the fresh tomato industry. Inter. Food Agribusiness Managem. Review. 1(13):45-64.

RAE. 2018. Real Academia Española Asociación de Academias de la Lengua Española. 2018. Diccionario de la real academia de la lengua española. Edición del tricentenario. https://dle.rae.es/?id=ZJ2KRZZ.

SIAP. 2014. Sistema de Información Agroalimentaria y Pesquera. Cultivos de agricultura protegida. Información estadística sobre cultivos, tipo de instalación, superficie cosechada y número de instalaciones. http://catalogo.datos.gob.mx/dataset/superficie-cubierta-ynumero-de-instalaciones-de-agricultura-protegida-siap.

SIAP. 2020. Sistema de Información Agroalimentaria y Pesquera. Agricultura protegida. https://www.gob.mx/siap/documentos/siacon-ng-161430.

SIAPRO. 2011. Sistema Nacional de Información de Agricultura Protegida. http://www.agriculturaprotegida.siap.gob.mx:8080/inver/map.phtml.

Ucan, C. I.; Sánchez Del Castillo, F.; Contreras, M. E. y Corona, S. T. 2005. Efecto de la densidad de población y raleo de frutos sobre el rendimiento y tamaño del fruto en tomate. Rev. Fitotec. Mex. 28(1):33-38. 
Valbuena-Díaz, N.; Leal-Guerra, M. y Urdaneta-Montiel, A. 2018. ADN organizacional y productividad en las empresas familiares. Desarrollo Gerencial. 10(1):105-122.

Valerio, C. D.; García, M. A.; Acero, de la C. R.; Castaldo, A.; Perea, J. M. y Martos, P. J. 2004. Metodología para la caracterización y tipificación de sistemas ganaderos. Departamento de producción animal Universidad de Córdoba. Producción animal y gestión. 9 p.

Vargas, C. J. M.; Palacios, R. M. I.; Camacho, V. J. H.; Aguilar, Á. J. y Ocampo, L. J. G. 2015. Factores de innovación en agricultura protegida en la región de Tulancingo, México. Rev. Mex. Cienc. Agríc. 6(4):827-840. 